\title{
Characterization of the hypothalamic transcriptome in response to food deprivation reveals global changes in long noncoding RNA, and cell cycle response genes
}

\author{
Hao Jiang ${ }^{2,5}$ - Thero Modise ${ }^{4} \cdot$ Richard Helm $^{2,4} \cdot$ Roderick V. Jensen ${ }^{3} \cdot$ \\ Deborah J. Good ${ }^{1,2,4}$ (D)
}

Received: 2 June 2015/Accepted: 5 October 2015/Published online: 16 October 2015

(c) Springer-Verlag Berlin Heidelberg 2015

\begin{abstract}
The hypothalamus integrates energy balance information from the periphery using different neuronal subtypes within each of the hypothalamic areas. However, the effects of prandial state on global mRNA, microRNA and long noncoding (lnc) RNA expression within the whole hypothalamus are largely unknown. In this study, mice were given either a 24-h fast, or ad libitum access to food. RNA samples were analyzed by microarray, and then a subset was confirmed using quantitative real-time PCR (QPCR). A total of 540 mRNAs were either up- or downregulated with food deprivation. Since gene ontology enrichment analyses identified several categories of mRNAs related to cell cycle processes, ten cell-cycle-related genes were further analyzed using QPCR with six confirmed to be significantly up-regulated and one downregulated in response to 24 -h fasting. While 22 independent microRNAs were differentially expressed by microarray,
\end{abstract}

Electronic supplementary material The online version of this article (doi:10.1007/s12263-015-0496-9) contains supplementary material, which is available to authorized users.

Deborah J. Good

goodd@vt.edu

1 Department of Human Nutrition Foods and Exercise, Virginia Tech, 1981 Kraft Drive (0913), Blacksburg, VA 24061, USA

2 Department of Biochemistry, Virginia Tech, Blacksburg, VA 24061, USA

3 Department of Biological Sciences, Virginia Tech, Blacksburg, VA 24061, USA

4 Program in Genomics, Bioinformatics and Computational Biology, Virginia Tech, Blacksburg, VA 24061, USA

5 Present Address: Department of Neurology, Washington University in St Louis, St Louis, MO 63110, USA secondary analysis by QPCR failed to confirm significant changes with fasting. There were 622 lncRNAs identified as differentially expressed, and of three tested by QPCR, two were confirmed. Overall, this is the first time that expression of hypothalamic lncRNAs has been shown to be responsive to food deprivation. In addition, this study is the first to identify a list of lncRNAs with high expression in RNA extracted from hypothalamus. Individual contributions from specific miRNA, lncRNA and mRNAs to the food deprivation response can now be further studied at the physiological and biochemical levels.

Keywords microRNA - lncRNA - mRNA · Fasting · Gene ontology $\cdot$ Hypothalamus

\section{Introduction}

The hypothalamus integrates central nervous system control of energy balance including responses to both food deprivation and re-feeding. Within the hypothalamus, multiple neuronal subtypes respond with both cellular signaling (for example, leptin and insulin signals) and gene regulatory responses involving protein-coding genes (mRNA), microRNAs (miRNA) and long noncoding RNAs (lncRNA). According to the Mouse Genome Informatics: Gene Expression Database, there are 2630 mRNAs with postnatal expression in the midbrain (http://www.infor matics.jax.org/, Smith et al. 2014). Of these, up to 588 are transcription factors (Good 2010). In addition, miRNAs also play a role in gene/protein regulation by repressing gene expression through an interaction with the mRNA transcript that results in either degradation or blocked translation of the mRNA. Finally, lncRNAs are emerging as key posttranscriptional regulators of mRNA, including 
regulation of transcription (both as scaffolds for chromatin modifying proteins and transcription factors, and as interfering moieties), splicing, mRNA decay and translation, as well as microRNA decoys (Rinn and Chang 2012; Yoon et al. 2013).

Differentially expressed hypothalamic mRNAs have been identified in selected rodent-based studies using the food deprivation/feeding paradigm. In a study by Poplawski et al. (2010), 48-h food deprivation in mice was used, in conjunction with a mouse expression array. This study found that global glucose metabolism was altered in response to this fast. In another study that uses laser microdissection in conjunction with rat whole-genome arrays, over 3000 differentially expressed mRNAs in the rat arcuate nucleus of the hypothalamus (Paulsen et al. 2009).

microRNAs have been reported to be differentially expressed in several brain disorders, including neuronal cancers, and neurodegeneration (Fassan et al. 2011; Roshan et al. 2009). There are also studies that demonstrate a role for microRNAs in more normal physiological processes, such as learning, synaptic plasticity and neuroadaptation (McNeill and Van Vactor 2012). A recent study using hypothalamic tissue from $o b / o b$ mice, a genetically obese and leptin-deficient strain has identified changes in miR200a, miR-200b and miR-429 (Crepin et al. 2014). Furthermore, within other tissue types, studies report an association of microRNA levels in adipose tissue, under high-fat diet or obesity, and in brown fat undergoing differentiation (Martinelli et al. 2010; Sun et al. 2011; Takanabe et al. 2008). Together, the evidence from these studies suggests that expression of hypothalamic microRNA may also change in response to normal changes in energy availability.

Long noncoding RNAs (lncRNA) are distinguished from microRNAs as they are more than 200 nucleotides in length (for reviews, see Kornfeld and Bruning 2014 and Kung et al. 2013). Similar to microRNAs, IncRNAs have been reported to be functionally associated with adipogenesis (Sun et al. 2013). In addition, studies also demonstrated that transcription of lncRNAs occurs in response to food supply and insulin/insulin-like growth factor levels (Ellis et al. 2014; Hellwig and Bass 2008). These indicate that IncRNAs might be involved in the energy balance regulation. In the brain, several papers have detailed the importance of lncRNA in neuronal differentiation and brain development (Aprea et al. 2013; Lin et al. 2014), but adult expression patterns under stress-type conditions such as fasting have not been reported.

The study reported herein utilized microarray platforms with the ability to capture exon-specific mRNA, microRNA and lncRNA data from the same samples. This allowed for full characterization of the whole hypothalamic transcriptome, including mRNA, microRNA and long noncoding RNA, in response to two prandial states, 24-h fasting and $a d l i b$ fed. As the global expression patterns have not been reported for either microRNA, nor for long noncoding RNAs in response to short-term fasting, these results characterize the simultaneous changes in all three subsets of the transcriptome, and help to further identify specific RNA targets in the fasting and fed response states.

\section{Experimental procedures}

\section{Animals}

The Institutional Animal Care and Use Committee at the Virginia Tech approved all studies. C57Bl/6 mice were purchased from The Jackson Laboratory as matched littermates, and only male mice were used in this experiment so that estrous cycles did not have to be taken into account during fasting. All mice were maintained under 12-h light/dark cycle with free access to food and water except as noted during experimentation. At the age of 8 weeks, mice were randomly separated to either a food-deprived ( $N=3$, microarray; $N=4-6$, QPCR) or ad lib group ( $N=3$, microarray; $N=4-6$, QPCR), and food was removed for deprived mice at 9 a.m. (Fig. 1a). After 24-h food deprivation, mice were euthanized and brains were collected. The hypothalamic region was isolated from the whole brain using a Zivic-Miller brain slicer, and taking a slice containing only the region between the optic chiasm and the mammillary bodies. That slice was further dissected to contain only the region between the cerebral peduncle on either side of the hypothalamus and to the top of where the third ventricle terminates, between the hypothalamus and thalamus. The slice was put into RNAlater $^{\circledR}$ solution (Ambion, USA) for RNA analysis. For a limited number of animals, gastrocnemius was collected to compare the sensitivity of our arrays for detecting tissuespecific microRNAs.

\section{RNA isolation}

The RNA was extracted using the mirVana ${ }^{\mathrm{TM}}$ PARIS $^{\mathrm{TM}}$ Kit (Ambion, USA) according to the manufacturer's procedure without modifications. Enrichment procedure for small RNAs was performed for microRNA study. RNA integrity was tested after extraction using Experion ${ }^{\mathrm{TM}}$ system (Bio-Rad, USA).

\section{Microarray and statistical analysis}

The microRNA analysis was done in triplicate $(N=3)$ using individual mouse samples and the Affymetrix 
Fig. 1 Experimental design and confirmation of food deprivation conditions. a Experimental design of the experiment, showing the timeline for the $a d l i b$ and fooddeprived groups of animals. b Serum glucose levels, relative to ad lib fed. c Serum leptin levels, relative to ad lib fed $* * P \leq 0.01$
A
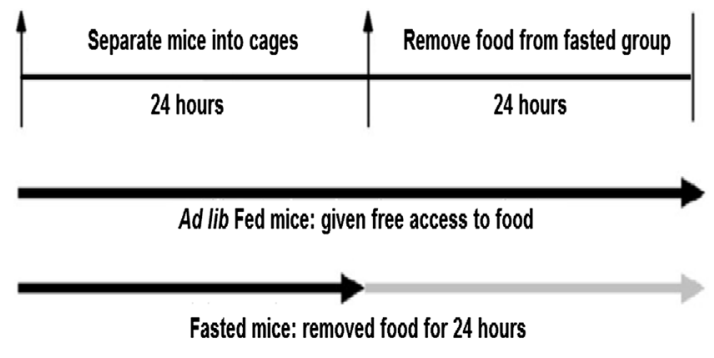

C

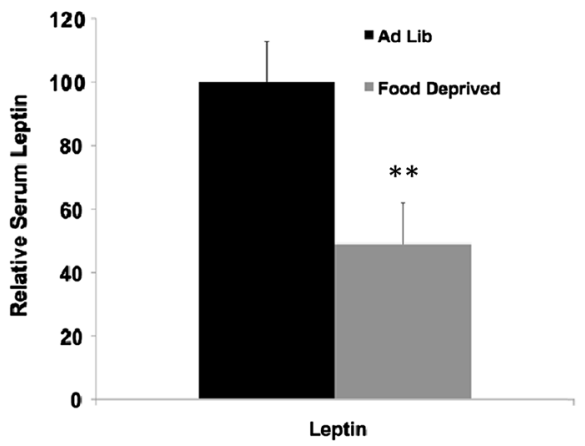

B 120

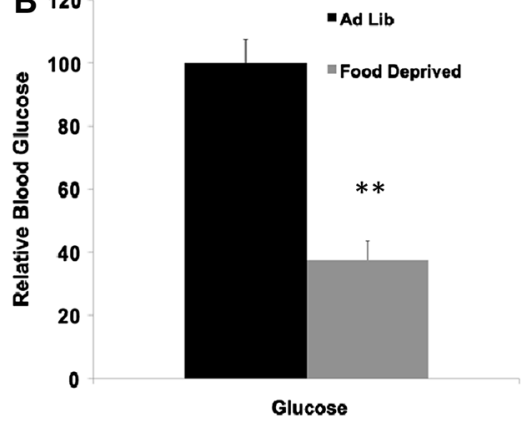

GeneChip ${ }^{\circledR}$ microRNA 1.0 array. The Affymetrix microRNA array raw data were extracted using the Affymetrix microRNA QC tool. Global normalization was used to normalize the raw data. The $\log 2$ values of the expression levels for each microRNA were processed, and Student's $t$ test was performed between $a d$ lib fed and food-deprived groups. To survey for candidate microRNA with differential expression, statistical criteria of $P$ value $\leq 0.05$ and fold change of $\geq 1.3$ were used to identify differentially expressed microRNAs.

mRNA analysis was performed in triplicate with the same individual mouse samples group as were analyzed for the microRNAs. These were done using the Affymetrix GeneChip ${ }^{\circledR}$ Mouse Exon 1.0 ST array. Global normalization was used to normalize the raw data. The $\log 2$ values of the expression levels for each mRNA were processed, and Student's $t$ test was performed between ad lib fed and fooddeprived groups. To survey for candidate mRNA with differential expression, statistical criteria used were a $P$ value $\leq 0.05$ and fold change of $\geq 1.3$.

Exon array-based lncRNA analysis was performed with the Affymetrix GeneChip ${ }^{\circledR}$ Mouse Exon 1.0 ST array data using Noncoder (Gellert et al. 2013), a Web interface designed for IncRNA analysis with the Affymetrix GeneChip ${ }^{\circledR}$ Mouse Exon 1.0 ST array. The CEL files used for mRNA analysis were uploaded to Noncoder, and data were processed using RMA normalization. The $\log 2$ value of the expression level for each IncRNA was processed, and the Student's $t$ test was performed between $a d$ lib fed and food-deprived groups. To survey for candidate lncRNA with differential expression, only lncRNAs with more than one probe set were used for further statistical comparisons, with cutoff of $P$ value $\leq 0.05$ and fold change of $\geq 1.3$.

\section{Quantitative PCR (QPCR) analysis}

To perform independent confirmation of statistically significant changes in transcript abundance, RNA from 4 to 6 additional mice was obtained for all confirmatory analyses, with microRNA expression levels measured utilizing the Taqman microRNA assay (Applied Biosystems, Foster City, CA). For each microRNA tested, 5 ng of small RNA-enriched samples from $N=4-6$ mice per assay was used. This number of individual mice used is based on experimental data and results from our laboratory and is sufficient to detect a 1.5-fold or better differences in expression (Vella et al. 2007). Reverse transcription and QPCR were performed according to the assay manual. The expression level of sno202 RNA was used as the normalization control in microRNA analysis, as this NC RNA has previously been shown to be most effective in a normalization analysis (Brattelid et al. 2011). In our hands, we also found sno-202 to be consistently expressed between tissues and conditions. All QPCR results were compared between groups using Student's $t$ test with $P$ value $\leq 0.05$ for statistical significance.

The expression levels of mRNA and IncRNA were measured using designed primers and the $\mathrm{iTaq}^{\mathrm{TM}} \mathrm{SYBR}^{\circledR}$ Green Supermix with Rox (Bio-Rad, Hercules, CA). New total hypothalamic RNA was isolated from $N=5-6$ mice, and $2 \mu \mathrm{g}$ of total RNA was treated with RQ1 RNase-Free DNase (Promega, Madison, Wisconsin) and then subjected 
to reverse transcription using M-MLV Reverse Transcriptase (Promega, Madison, Wisconsin). A $20 \mathrm{ng}$ aliquot of cDNA was then used for QPCR analysis using the ABI 7900 system (Applied Biosystems, Foster City, CA). The expression level of $\beta$-actin was used as the normalization control. All QPCR results were compared between groups using Student's $t$ test with $P$ value $\leq 0.05$ for statistical significance. For lncRNA analysis, QPCR products were then sequenced to confirm that the sequence of the amplicon was unique to the lncRNA region.

\section{Leptin and Glucose measurement}

Whole blood was collected immediately after each mouse was euthanized. Blood glucose level was measured using FreeStyle Freedom Lite ${ }^{\circledR}$ Blood Glucose Monitoring System (Abbott Laboratories, USA). The serum was collected from the whole blood sample by centrifuging at $1000 \times g$ for $10 \mathrm{~min}$ at $4{ }^{\circ} \mathrm{C}$. Serum leptin level was measured using the Mouse Leptin Quantikine ELISA kit (R\&D System, USA) according to the manufacturer's instructions and using manufacturer-provided standards to produce the standard curve. Blood glucose and leptin level were compared between groups using Student's $t$ test with $P$ value $\geq 0.05$ for statistical significance.

\section{GO and STRING analysis}

Gene ontology analysis was performed using GeneCodis3 (Carmona-Saez et al. 2007; Nogales-Cadenas et al. 2009; Tabas-Madrid et al. 2012). Genes passing the cutoff of microarray analysis were used for the GO Biological Process, GO Molecular Function, GO Cellular Component and KEGG pathways analysis. For the settings of statistical parameters, the minimum number of genes was set to two, and a hypergeometric statistical test and FDR $P$ value correction were used. The results were listed with the multiple-testing-corrected, hypergeometric $P$ value.

STRING version 10 was used in its online format to generate an interactive network mode using the all cell cycle genes tested for QPCR (Franceschini et al. 2013). The confidence view was used with one expansion of the protein interactions network.

\section{Results}

\section{Leptin and glucose levels}

Following 24-h food deprivation (Fig. 1a), serum leptin and blood glucose levels for each treatment group showed a significant reduction, as would be expected from fasting conditions (Fig. 1b, c).

\section{Overall transcriptome changes}

Twenty-four-hour food deprivation resulted in changes in the overall hypothalamic transcription of varying magnitude. Using DNA microarrays and the statistical selection criteria of a $P$ value of $\leq 0.05$ and fold change of $\geq 1.3$, a subset of the candidate differentially expressed genes was identified. As shown in Table 1, the majority of detectable changes were found within the mRNA and IncRNA transcriptome, while the fewest changes were found within the microRNA transcription.

\section{mRNA microarray analysis and QPCR validation}

The data from the Affymetrix exon arrays (Supplemental Data File 1, excel list) were first used to identify differentially expressed hypothalamic mRNAs between ad lib fed and 24-h food-deprived animals. The $\log 2$ scatter plot comparison (Fig. 2a) suggested that there is no dramatic transcriptome adjustment in response to food deprivation, while volcano plot analysis (Fig. 2b) indicated that there were still some significant changes between the two conditions. In all, there were 540 candidate differentially expressed mRNAs identified, with 298 mRNAs found to be upregulated and 242 mRNAs down-regulated with food deprivation (Table 1). The up- and down-regulated mRNAs identified using the array data are shown in Supplemental Table 1. The top ten mRNAs with the highest hypothalamic expression levels overall are shown in Supplemental Table 2. None of differentially expressed mRNAs were in the list of those with the highest hypothalamic expression. A subset of the differentially expressed candidate genes was selected for further analysis based on probable role in energy balance regulation, or brain function, and $P$ value. As shown
Table 1 Comparison of microRNA, lncRNA and mRNA array results

\begin{tabular}{lrrr}
\hline & microRNA & lncRNA & mRNA \\
\hline Total detected & 536 & 12,521 & 16,294 \\
Number of those significantly up-regulated by fasting & 16 & 421 & 298 \\
Number of those significantly down-regulated by fasting & 6 & 201 & 242 \\
\hline
\end{tabular}

Total detected RNA species, and those found to be significantly up- or down-regulated by food deprivation, compared to ad lib feeding, based on the criteria of at least 1.3-fold (either direction) different, and $P \leq 0.05$ 

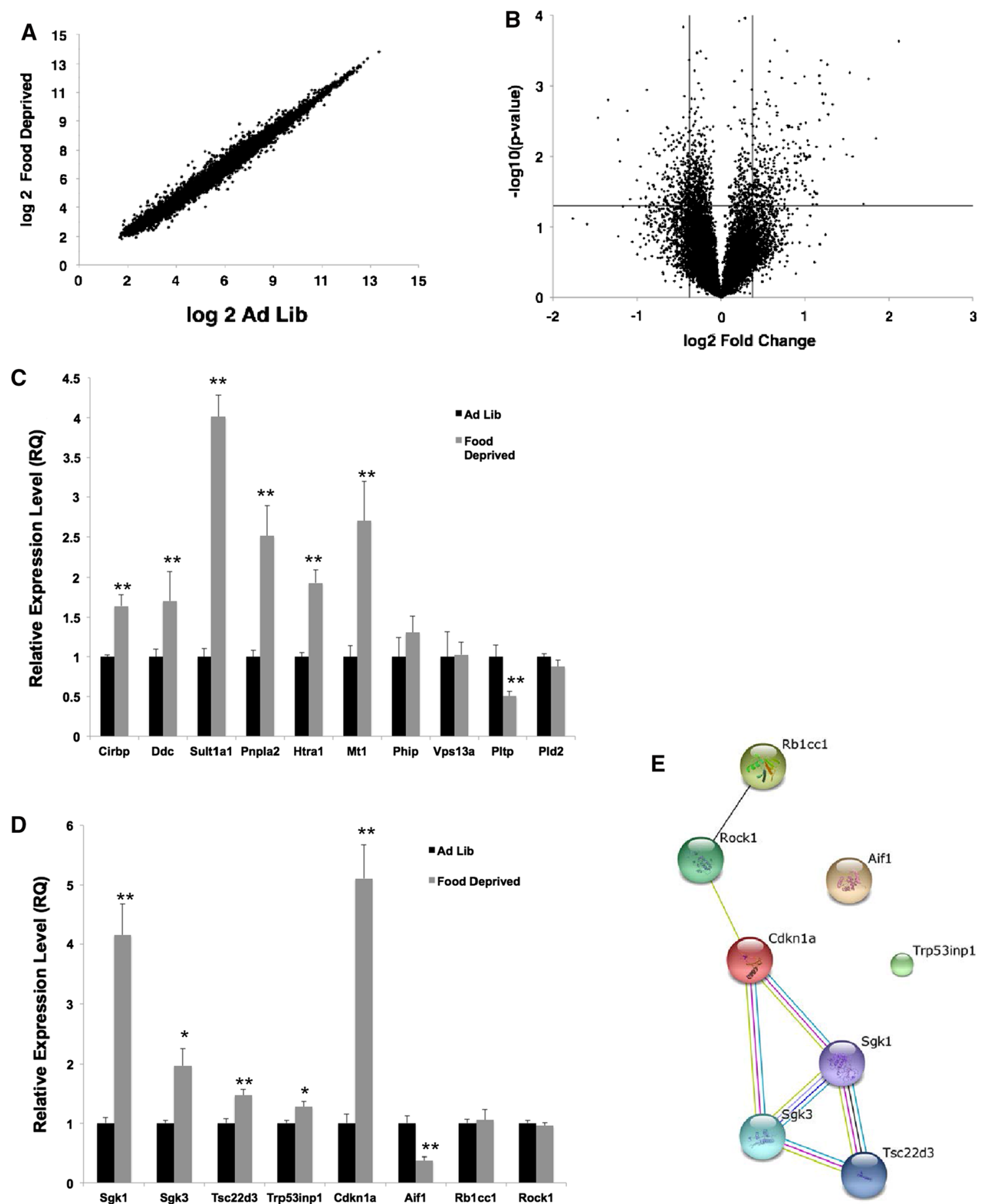

Fig. 2 mRNA array plots and QPCR analysis. a $\log 2$ scatter plot of hypothalamus mRNA array data from Affymetrix array. Data were filtered using a $P$ value $<0.05$ and 1.3 -fold change. b Volcano plot showing $P$ value versus $\log 2$ expression level for data from Affymetrix array. Lines indicate where significance cutoff values were made. c QPCR analysis of selected mRNAs that were differentially expressed in food-deprived versus $a d$ lib mice. $\mathbf{d}$ mRNAs were selected from cell

in Fig. 2c, d, most genes chosen using these criteria were confirmed to be significantly differentially expressed in independent samples isolated from $a d l i b$ and food-deprived cycle GO categories, and their expression levels tested by QPCR using $N=5-6$ new samples for each group. All mRNA levels are reported as relative to levels in ad lib fed animals and normalized to the housekeeping gene $\beta$-actin. e String v10 was used to generate a network using the proteins tested in 7A as input. The network (interactive network mode) was expanded by one level. Only the names of the proteins input are shown. $* P \leq 0.05 ; * * P \leq 0.01$

animals. Of ten genes selected as differentially regulated with 24-h fasting, seven of these changes were confirmed by QPCR using an independent test set of animals. 
Gene ontology (GO) analysis of the 540 differentially regulated candidate genes between $a d$ lib fed and fooddeprived mice was performed, and the top five GO terms for each of the major component or process category are shown in Table 2. Close examination of the entire dataset of significant categories $(P$ value $\leq 0.05)$ revealed that cell-cyclerelated categories were found once in the top five list, and then multiple times in the remaining significant categories. These additional categories of cell-cycle-related terms are shown in Table 3. A subset of genes found in cell cycle categories was further analyzed using QPCR. Of eight cell cycle category genes analyzed, six were confirmed to be significantly differentially regulated in new independent hypothalamic mRNA samples from $a d$ lib and food-deprived animals (Fig. 2e). Using STRING (Search Tool for Retrieval of Interacting Genes/proteins) (Szklarczyk et al. 2011) analysis, four of these comprise a network of protein products involved in cell cycle control, and all of the four, except rock 1, showed significantly different expression by QPCR (Fig. 2f) using the independent test set.

\section{microRNA microarray analysis and QPCR validation}

microRNA expression analysis was performed in triplicate on the Affymetrix microRNA microarray platforms (Supplemental Data File 2, excel list). Scatter plot analysis showed that the overall expression level differences between the 24-h fasting and ad lib treatment groups were similar and suggestive of few differentially regulated microRNAs, especially within the significance criteria (Fig. 3a, b). A total of 536 microRNAs were detected with the microarrays, but only 22 candidates for differential expression were identified using the relatively loose statistical criteria of $P \leq 0.05$ and fold change $\geq 1.3$, with 16 up-regulated and 6 down-regulated (Table 1 and Supplemental Table 3). A subset of these candidate microRNAs was chosen for further analysis based on $p$ value and whether they had been found to be involved in any aspect of energy balance in previous studies, including those using other tissues than brain. These criteria identified six for

Table 2 GO analysis (mRNA arrays)

\begin{tabular}{|c|c|c|c|}
\hline GO term & Gene count (significant) & Gene count (reference) & $P$ value \\
\hline \multicolumn{4}{|l|}{ Biological process } \\
\hline GO:0006810: transport & 56 & 1620 & $8.73 \mathrm{E}-07$ \\
\hline GO:0007049: cell cycle & 26 & 533 & $3.41 \mathrm{E}-05$ \\
\hline GO:0016310: phosphorylation & 29 & 689 & $8.99 \mathrm{E}-05$ \\
\hline GO:0048812: neuron projection morphogenesis & 7 & 36 & 0.000192 \\
\hline GO:0006974: response to DNA damage stimulus & 17 & 308 & 0.000447 \\
\hline \multicolumn{4}{|l|}{ Cellular component } \\
\hline GO:0016020: membrane & 178 & 5723 & $4.76 \mathrm{E}-24$ \\
\hline GO:0005886: plasma membrane & 103 & 2782 & $1.23 \mathrm{E}-17$ \\
\hline GO:0005634: nucleus & 144 & 4867 & $7.00 \mathrm{E}-17$ \\
\hline GO:0005737: cytoplasm & 141 & 5026 & $1.33 \mathrm{E}-14$ \\
\hline GO:0016021: integral to membrane & 143 & 5398 & $6.95 \mathrm{E}-13$ \\
\hline \multicolumn{4}{|l|}{ Molecular function } \\
\hline GO:0005515: protein binding & 100 & 2999 & $3.92 \mathrm{E}-16$ \\
\hline GO:0046872: metal ion binding & 91 & 2802 & $5.08 \mathrm{E}-14$ \\
\hline GO:0000166: nucleotide binding & 64 & 1999 & $8.20 \mathrm{E}-10$ \\
\hline GO:0005524: ATP binding & 47 & 1421 & $6.59 \mathrm{E}-08$ \\
\hline GO:0003677: DNA binding & 51 & 1635 & $1.10 \mathrm{E}-07$ \\
\hline \multicolumn{4}{|l|}{ KEGG pathways } \\
\hline KEGG:04670: leukocyte transendothelial migration & 9 & 117 & $4.27 \mathrm{E}-05$ \\
\hline KEGG:04724: glutamatergic synapse & 9 & 123 & $6.32 \mathrm{E}-05$ \\
\hline KEGG:04010: MAPK signaling pathway & 10 & 263 & 0.004306 \\
\hline KEGG:04144: endocytosis & 9 & 213 & 0.003344 \\
\hline KEGG:04062: chemokine signaling pathway & 12 & 177 & $8.61 \mathrm{E}-06$ \\
\hline
\end{tabular}

All significantly differentially expressed mRNAs (529 total) were used in this analysis. The top five GO terms for each category are shown. The number of genes significant for that category (gene count, significant), the number of total genes in the reference category (gene count, reference) and $P$ value for the GO term are shown 
Table 3 Cell cycle category GO terms

\begin{tabular}{lcrr}
\hline GO term & Gene count (significant) & Gene count (reference) & $P$ value \\
\hline Cell cycle category & & 26 & 533 \\
GO:0007049: cell cycle (BP) & 7 & 48 & $3.41 \mathrm{E}-05$ \\
GO:0001938: positive regulation of endothelial cell proliferation (BP) & 2 & 4 & 0.000652 \\
GO:0001936: regulation of endothelial cell proliferation (BP) & 3 & 14 & 0.031983 \\
GO:0050768: negative regulation of neurogenesis (BP) & 14 & 340 & 0.029825 \\
GO:0043066: negative regulation of apoptotic process (BP) & 16 & 504 & 0.017967 \\
GO:0006915: apoptotic process (BP) & & 0.043501 \\
\hline
\end{tabular}

List of all significant GO terms related to cell cycle categories is shown. The number of genes significant for that category (gene count, significant), the number of total genes in the reference category (gene count, reference) and $P$ value for the GO term are shown
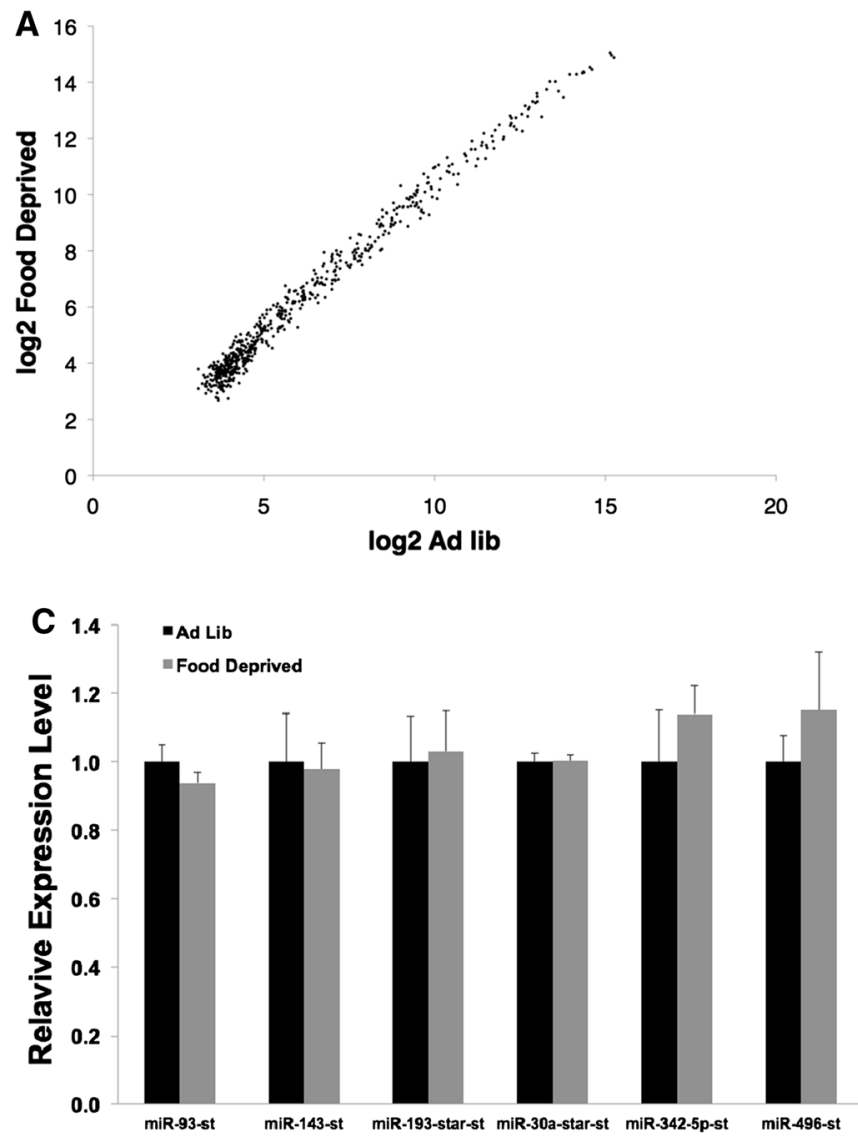

Fig. 3 microRNA array plots and QPCR analysis. a $\log 2$ scatter plot of hypothalamus microRNA array data from Affymetrix array. Data were filtered using a $P$ value $<0.05$ and 1.3 -fold change. b Volcano plot showing $P$ value versus $\log 2$ expression level for data from Affymetrix array. Lines indicate where significance cutoff values were made. c microRNA QPCR analysis in hypothalamic tissue of ad

further expression analysis using independent samples. However, QPCR analysis failed to confirm that any of these chosen microRNAs were significantly different in an independent analysis between $a d l i b$ and food-deprived
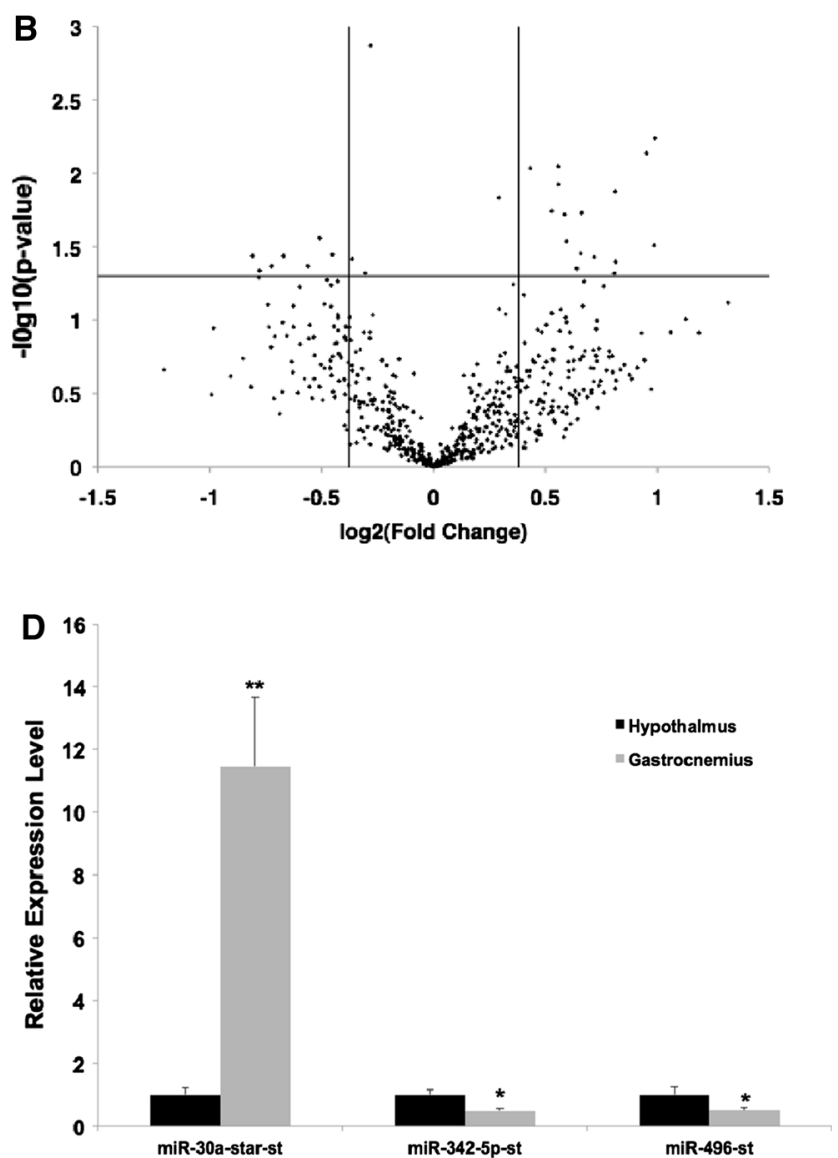

$l i b$ versus food-deprived mice. microRNAs were selected using the cutoff criteria, and expression level tested in $N=4-6$ new samples for each group. All microRNA levels are reported relative to levels in ad lib fed animals and normalized to the sno-202 microRNA. d microRNA expression in skeletal muscle versus hypothalamus. $* P \leq 0.05 ; * * P \leq 0.01$

treatment groups (Fig. 3c). Nevertheless, differences could be detected comparing skeletal muscle versus hypothalamic microRNA QPCR for three of these microRNAs (Fig. 3d), indicating that detection should have been 
possible with these probe sets had differences existed. For additional verification of the system, ad lib fed mice were used to compare hypothalamic and skeletal muscle (gastrocnemius) microRNAs (Supplemental Figure 1A), revealing the exploded comet shape suggestive of the expected significant differences in expression levels of specific microRNAs between the two tissue types. Specific microRNAs were then analyzed by QPCR using total RNA from both hypothalamus and skeletal muscle of both ad lib and fasted mice. As shown in Supplemental Fig. 1b, let-7d was highly expressed in both tissues, with similar expression levels under all conditions. Expression of the skeletal muscle-specific microRNA, miR-1, was significantly higher in skeletal muscle than in hypothalamus tissue, in both $a d$ lib and fasted mice, as expected (Supplemental Figure 1C). Conversely, the nervous system-specific microRNA, miR-124a, was highly expressed in hypothalamic RNA isolated from both fed and fasted mice, when compared to its detection at a significantly lower level in gastrocnemius RNA isolated from either mice in either treatment group (Supplemental Figure 1D). The 10 highest expressing hypothalamic microRNAs in ad lib fed mice are shown in Supplemental Table 4. None of the microRNAs with the highest hypothalamic expression showed evidence of differential expression, but these results provide information on the hypothalamic microRNAome, overall, with highly expressed microRNAs warranting future exploration into their roles.

\section{IncRNA microarray analysis and QPCR validation}

The Affymetrix exon arrays were also used to identify hypothalamic lncRNAs which were differentially expressed between ad lib fed and 24-h fasted animals. Only IncRNAs with two or more probe sets were used in the analysis, giving a total of $12,521 \operatorname{lncRNAs}$ that could be detected by Noncoder (Table 1) (Supplemental Data File 3, excel list) (Gellert et al. 2013). Scatter plot analysis showed that the overall expression level differences between fooddeprived and ad lib treatment groups were similar. Employing the statistical criteria of $P \leq 0.05$ and a fold change of $\geq 1.3,421$ candidate lncRNAs were found to be up-regulated by 24 -h fasting compared to ad lib feeding, whereas 201 lncRNAs were down-regulated (Table 1; Fig. 4a, b). A list of the differentially expressed lncRNAs is shown in Supplemental Table 5. Three of the candidate lncRNAs with the highest fold change accompanied by highly significant $p$ values between $a d$ lib and food-deprived groups were selected for further analysis by QPCR. As shown in Fig. 4c, two lncRNAs, AK038506 and AK049914, were confirmed to be significantly differentially expressed, both on the array platform, and using QPCR in independent samples isolated from $a d$ lib and food-deprived animals. The top ten lncRNAs with the highest hypothalamic expression levels overall are shown in Supplemental Table 6. Similar to the microRNAs, none of the lncRNAs with the highest hypothalamic expression showed evidence of differential expression, but provide information on the hypothalamic lncRNAome, and again those that might warrant further exploration.

\section{Discussion}

The results of this study yield two major findings. First, after a 24-h fast, the hypothalamic microRNA response is minimal, when compared to the lncRNA or mRNA response. Statistically significant changes in microRNA expression levels could not be confirmed by QPCR in an independent test set, and to date, this is the only global hypothalamic analysis of the microRNA transcriptome in response to food deprivation. Second, and in contrast to the microRNA results, there are multiple lncRNAs and mRNAs differentially expressed following food deprivation. While the lncRNAs are not well characterized, and pathway analysis is not available for this subset of RNA, the mRNAs dataset revealed a high number of differentially expressed mRNAs within the cell cycle categories following gene ontology analysis. As noted, multiple pathways, including phosphorylation, stress response and specific enzyme categories, are also significantly changed when the mRNA datasets were compared between these conditions. This is the first study to fully characterize the transcriptome of the hypothalamus in 24-h fasting, compared to the ad lib fed animals.

Given the previous published studies showing differential regulation of microRNAs in brain, adipose and other tissues conditions such as cancer or obesity, it was surprising to find so few differentially expressed microRNAs via microarray analysis and none that could be confirmed by further QPCR analysis (Fassan et al. 2011; Martinelli et al. 2010; McNeill and Van Vactor 2012; Roshan et al. 2009; Sun et al. 2011; Takanabe et al. 2008). Although current database information suggests that there are only 105 microRNAs expressed in the midbrain above the detection threshold (http://www.microrna.org), we detected 536 microRNAs using the Affymetrix microarrays. Our data confirmed that the let-7 family, miR-124a, miR-125 family and miR-138 were highly represented in the hypothalamic microRNAome, and found several others such as miR-709 and miR-690 which have not previously been shown to be highly expressed in this region (Bak et al. 2008; Meister et al. 2013; Olsen et al. 2009). Moreover, we were able to demonstrate that the microarrays could detect many statistically significant differences between hypothalamus and skeletal muscle. However, only 22 

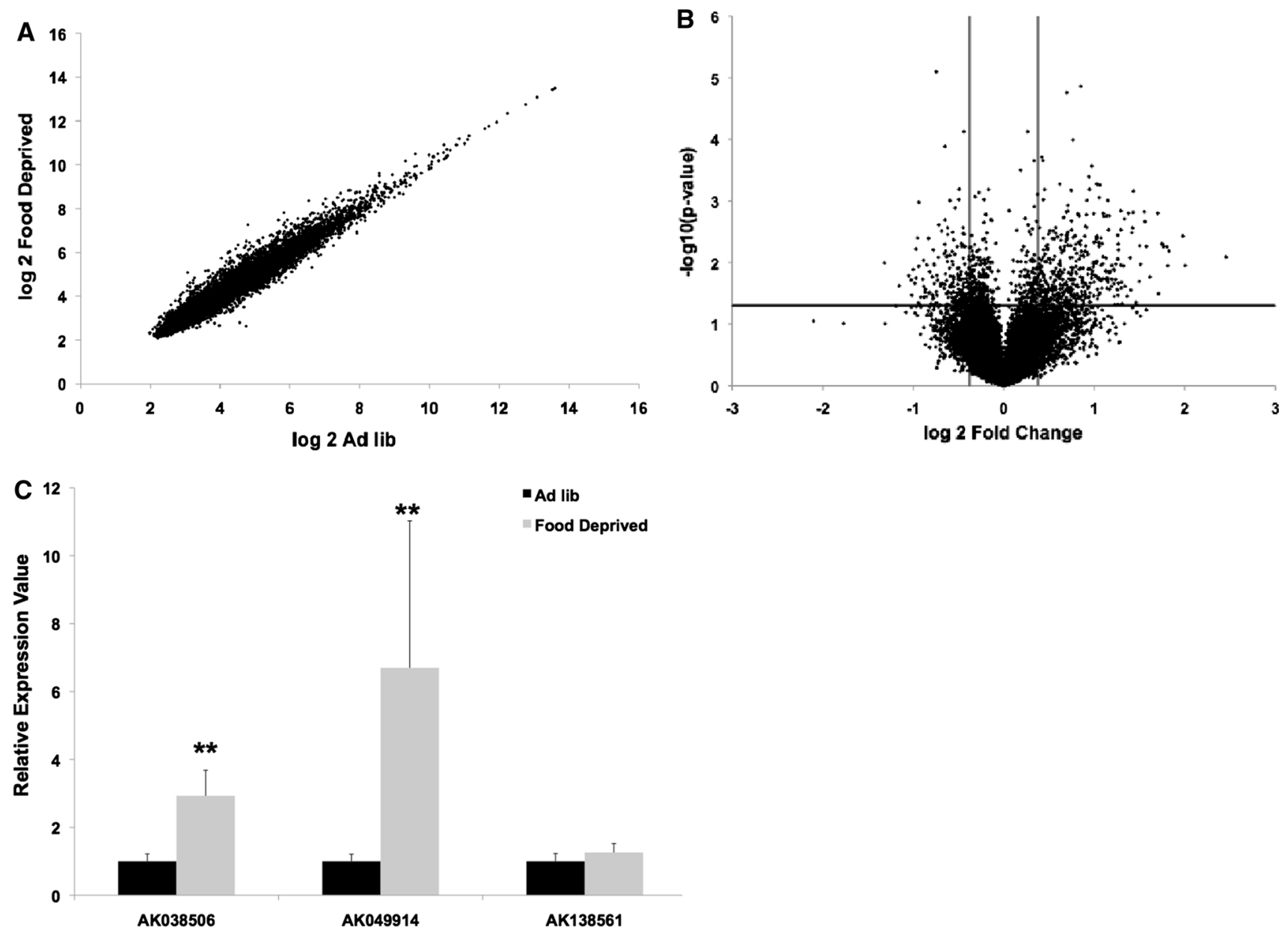

Fig. 4 lncRNA array plots and QPCR analysis. a $\log 2$ scatter plot of hypothalamus lncRNA array data. Data were filtered using a $P$ value $\leq 0.05$ and 1.3 -fold change. b Volcano plot showing $P$ value versus $\log 2$ expression level for data from lncRNA results. Lines indicate

where significance cutoff values were made. c lncRNA QPCR analysis using hypothalamic tissue of $a d$ lib versus food-deprived mice. All lncRNA levels are reported relative to levels in ad lib fed animals and normalized to the sno-microRNA $* * P \leq 0.01$

candidate differentially expressed microRNAs were identified between the 24-h fasted and ad lib fed states in the hypothalamus, and none of the changes were confirmed to be statistically significant in an independent set of samples using QPCR. In a study by Sangiao-Alvarellos and colleagues which examined hypothalamic miRNAs in highfat-fed, versus chronically restricted, low-fat-fed animals (fed at $65 \%$ of control, but not completely food restricted), ten differentially expressed miRNAs were identified (Sangiao-Alvarellos et al. 2014). However, only one of these significant differences was repeatable in a later figure of the same paper with 48-h fast (Sangiao-Alvarellos et al. 2014). None of the microRNAs from Sangiao-Alvarellos and colleagues were differentially expressed in our samples, and thus, the findings by Sangiao-Alvarellos and colleagues are therefore consistent with ours, in few hypothalamic miRNAs changing in response to negative energy balance.
It is possible that statistically significant miRNA expression may have been detected at earlier time points but this beyond the scope of the current study. In addition, as this study examined the transcriptome of the whole hypothalamus, rather than region-specific transcriptomes, the possibility exists that region-specific changes were missed. For example, deletion of the dicer gene within hypothalamic POMC neurons was shown to lead to obesity in adulthood (Schneeberger et al. 2012). Given this finding and the dicer's global role in processing microRNAs, it is likely that examining of nuclei-specific microRNAs may still reveal differential expression patterns with fasting or other conditions that change energy availability in the body.

Hypothalamic IncRNAs have not been previously implicated in any condition involving energy availability, making this study the first to both characterize the top differentially regulated IncRNAs and provide a list of 
highly expressed lncRNAs for this tissue. Noncoding RNAs, such as lncRNA, are of increasing interest, having predicted roles in transcriptional regulation during brain development and disease (Clark and Blackshaw 2014; Qureshi and Mehler 2013). This study identified more than 12,000 lncRNAs that are expressed within whole hypothalamus and is the first of its kind to do so for mouse hypothalamus undergoing ad lib feeding or fasting. To date, only one other study in canines has examined hypothalamic IncRNAs, and this was done in nonfasting male beagles (Roy et al. 2013). The study identified 57 lncRNAs in the canine hypothalamus, although none appear to have any overlap with those found in this study using our criteria. Other studies, not using hypothalamic tissue, have implicated lncRNAs in various nutritional states, including those found in the gut in germ free, versus microbiota (free-living) conditions, and those identified in diabetics (SNP association, without verification, Liang et al. 2015; Wessel et al. 2015). Additionally, lncRNAs have been shown to enhance brown and white adipocyte differentiation, participate in pancreatic beta-cell function and possibly protect against diet-induced obesity, as shown for SRA, the steroid receptor RNA activator (Kameswaran and Kaestner, 2014; Liu et al. 2014; Sun et al. 2011; Sun et al. 2013; Xu et al. 2015; You et al. 2015). The most relevant to our work would be SRA, as a whole body knockout of this IncRNA protected against diet-induced obesity (Liu et al. 2014). However, our results confirm theirs showing low expression in hypothalamus, with SRA not being found differentially expressed in our results.

Of the more than 12,000 identified, more than 600 lncRNAs were found differentially expressed by microarray analysis and can now be further studied by QPCR or other methods their possible roles in energy balance. Of the two lncRNAs confirmed by QPCR analysis to be differentially expressed with fasting, neither has been previously characterized for any tissue or condition. AK038506, which we have found to be significantly increased with fasting, was originally identified in adult male mouse hypothalamic cDNA during global cloning studies (Carninci et al. 2005). It is a 1933-nucleotide sequence with only minimal (less than $5 \%$ overlap) to other GeneBank sequences by nucleotide BLAST (data not shown). AK049914, which is also significantly increased with fasting, is a 4061-nucleotide sequence, originally found in adult male mouse hippocampus cDNA, and sequence analysis indicates that this lncRNA appears to overlap within the third and fourth exons of the apolipoprotein D gene (Apod) (Carninci et al. 2005). Furthermore, there is a 289 bp sequence (NC_000082.6: 31308226 to 31308521 ) that has a very high similarity ( $97 \%$ identities based on the BLAST search) to a sequence localized about $200 \mathrm{bp}$ upstream to itself (NC_000082.6: 31309821 to 31310117), but still within the two exons of Apod. These together indicate a potential link of transcription activities between AK049914 and Apod. In fact, it has been reported that Apod is up-regulated by calorie restriction in whole brain, skeletal muscle and heart (Yan et al. 2012). A study from 1994 linked the apolipoprotein D gene to obesity and fasting insulin status (Vijayaraghavan et al. 1994). A more recent study has explored the finding that several Alzheimer's disease-associated genes, including apolipoprotein E, have IncRNAs within their genes (Holden et al. 2013). These findings are consistent with the up-regulation of AK049914 during fasting and open the door for future studies on the role of IncRNAs in energy balance regulation, hypothalamus function and diseases such as Alzheimer's disease.

In this study, animals were food-deprived for $24 \mathrm{~h}$, in order to analyze the effects of food deprivation on the whole transcriptome. The mRNAs were studied as part of the whole transcriptome, with interesting findings detected in cell-cycle-related gene expression, through both GO and STRING network analyses. In particular, p21/Cdkn1a was one of the most significant food deprivation up-regulated genes, and this finding was confirmed by QPCR. p21/Cdkn1a controls progression of cells through the cell cycle, by blocking cyclin-dependent kinase activity, with two published reports linking p21/Cdkn1a activity, obesity and adipose tissue (Inoue et al. 2008; Nakatsuka et al. 2012). Interestingly, the link between fasting-induced up-regulation of $\mathrm{p} 21 / \mathrm{Cdkn} 1 \mathrm{a}$ has previously been made for hypothalamic tissue as well. In a 2010 study of C57B1/6 mice undergoing a 48-h food deprivation, p21/Cdkn1a expression was significantly up-regulated (Poplawski et al. 2010). More recently, in a 2013 paper, liver and hypothalamus of mice fasted 24 and $48 \mathrm{~h}$ showed significant induction of a $\mathrm{p} 21 / \mathrm{Cdkn} 1 \mathrm{a}$ promoter construct (Tinkum et al. 2013). Further examination of our dataset revealed that there were many genes with GO categories related to cell cycle processes. Based on this, we looked into whether differential regulation of other cell-cycle-related genes could be confirmed by QPCR and were able to confirm six out of eight of these genes in follow-up studies on new tissue samples. The apparent relationship between fasting and cell cycle regulation by this dataset is an interesting one, given that studies suggest that the onset of hypothalamic adult neurogenesis is related to high levels of leptin or ciliary neurotrophic factor (Kokoeva et al. 2005). However, up-regulation of p21/Cdkn1a by food deprivation, which lowers serum leptin levels, is consistent with a blockage in cell cycle and reduced proliferation and the opposite of what is seen with leptin and/or CNTF exposure (Cheng 2013). At this time, more studies would be needed to determine whether differential regulation of the p21/ Cdkn1a and other cell cycle regulation-associated genes 
result in apoptosis, cell cycle arrest or some other event following food deprivation.

It is known, through work using several different models, that calorie restriction can increase individual longevity, and at least one study has examined hypothalamic gene expression in response to a $60 \%$ restricted diet (Fu et al. 2006). In that study, nine genes were differentially regulated by calorie restriction in aging mice, but none overlapped with those in our study. Calorie restriction can reduce levels of oxidative stress and protect proteins, lipids and DNA form oxidative damage, thereby possibly contributing to cellular protection against diabetes, cardiovascular disease, cancers and neurodegenerative diseases (Mattson 2005; Mattson and Wan 2005; Sohal and Weindruch 1996). While an acute fast, such as 24-h food deprivation, is a different treatment than longterm calorie restriction, biological processes category such as "response to DNA damage stimulus" (GO:0006974), "oxidation-reduction process" (GO:0055114) and "lipid biosynthetic process" (GO:0008610) was found among the top groups of GO terms. Likewise, stress-related mRNAs such as Sult1a1, Cirbp and Tsc22d3 were also confirmed to be differentially regulated by QPCR (De Leeuw et al. 2007; Maglich et al. 2004; Szklarczyk et al. 2012). Our findings present new directions to study the potential relationship between the acute and chronic effects of calorie restriction.

In summary, there is little evidence of a global microRNA response following food deprivation of $24 \mathrm{~h}$ in mouse hypothalamic tissue, even though microRNAs were detectable in hypothalamus and those tested were differentially expressed when compared in skeletal muscle. It is possible that physiological stressors other than food deprivation may result in differential microRNA expression, or the use of whole hypothalamus in our analysis was not appropriate to detect the select, nuclei-specific microRNAs that were differentially expressed following food deprivation. The study, however, was designed to fully characterize the whole transcriptome, including lncRNAs, of which over 600 found to be differentially expressed. In addition, close to $50 \%$ of all of the mRNA species represented on the array were also detectable in hypothalamic tissue, and over 500 of them were differentially regulated in response to food deprivation. Our confirmation of a relationship between food deprivation and the differential regulation of mRNAs within the cell cycle control gene ontology categories now provides new insight into hypothalamic plasticity and adult hypothalamic neurogenesis (Kokoeva et al. 2005). These data also suggest new directions of research aimed at clarifying role of lncRNA in the fields of obesity, caloric restriction and nutrigenomics, specifically in identifying possible hypothalamic gene regulatory pathways activated by food deprivation signals.
Acknowledgments This work was supported by a Grant from the National Institutes of Health (DK086655, DJG, RH, and RVJ, coPIs). We thank Jinhua Zhang for excellent technical assistance, and members of the Animal Care Staff in the Integrated Life Sciences Building for providing exceptional animal care.

\section{Compliance with ethical standards}

Conflict of interest Hao Jiang, Thero Modise, Richard Helm, Roderick V. Jensen and Deborah J. Good declare that they have no conflict of interest.

Ethical standard All institutional and national guidelines for the care and use of laboratory animals were followed.

\section{References}

Aprea J, Prenninger S, Dori M, Ghosh T, Monasor LS, Wessendorf E, Zocher S, Massalini S, Alexopoulou D, Lesche M, Dahl A, Groszer M, Hiller M, Calegari F (2013) Transcriptome sequencing during mouse brain development identifies long non-coding RNAs functionally involved in neurogenic commitment. EMBO J 32(24):3145-3160. doi:10.1038/emboj.2013.245

Bak M, Silahtaroglu A, Moller M, Christensen M, Rath MF, Skryabin B, Tommerup N, Kauppinen S (2008) MicroRNA expression in the adult mouse central nervous system. RNA. doi:10.1261/rna. 783108

Brattelid T, Aarnes EK, Helgeland E, Guvaag S, Eichele H, Jonassen AK (2011) Normalization strategy is critical for the outcome of miRNA expression analyses in the rat heart. Physiol Genom. doi:10.1152/physiolgenomics.00131.2010

Carmona-Saez P, Chagoyen M, Tirado F, Carazo JM, PascualMontano A (2007) GENECODIS: a web-based tool for finding significant concurrent annotations in gene lists. Genome Biol. doi:10.1186/gb-2007-8-1-r3

Carninci P, Kasukawa T, Katayama S, Gough J, Frith MC, Maeda N, Oyama R, Ravasi T, Lenhard B, Wells C, Kodzius R, Shimokawa K, Bajic VB, Brenner SE, Batalov S, Forrest AR, Zavolan M, Davis MJ, Wilming LG, Aidinis V, Allen JE, Ambesi-Impiombato A, Apweiler R, Aturaliya RN, Bailey TL, Bansal M, Baxter L, Beisel KW, Bersano T, Bono H, Chalk AM, Chiu KP, Choudhary V, Christoffels A, Clutterbuck DR, Crowe ML, Dalla E, Dalrymple BP, de Bono B, Della Gatta G, di Bernardo D, Down T, Engstrom P, Fagiolini M, Faulkner G, Fletcher CF, Fukushima T, Furuno M, Futaki S, Gariboldi M, Georgii-Hemming P, Gingeras TR, Gojobori T, Green RE, Gustincich S, Harbers M, Hayashi Y, Hensch TK, Hirokawa N, Hill D, Huminiecki L, Iacono M, Ikeo K, Iwama A, Ishikawa T, Jakt M, Kanapin A, Katoh M, Kawasawa Y, Kelso J, Kitamura H, Kitano H, Kollias G, Krishnan SP, Kruger A, Kummerfeld SK, Kurochkin IV, Lareau LF, Lazarevic D, Lipovich L, Liu J, Liuni S, McWilliam S, Madan Babu M, Madera M, Marchionni L, Matsuda H, Matsuzawa S, Miki H, Mignone F, Miyake S, Morris K, Mottagui-Tabar S, Mulder N, Nakano N, Nakauchi H, $\mathrm{Ng}$ P, Nilsson R, Nishiguchi S, Nishikawa S, Nori F, Ohara O, Okazaki Y, Orlando V, Pang KC, Pavan WJ, Pavesi G, Pesole G, Petrovsky N, Piazza S, Reed J, Reid JF, Ring BZ, Ringwald M, Rost B, Ruan Y, Salzberg SL, Sandelin A, Schneider C, Schonbach C, Sekiguchi K, Semple CA, Seno S, Sessa L, Sheng Y, Shibata Y, Shimada H, Shimada K, Silva D, Sinclair B, Sperling S, Stupka E, Sugiura K, Sultana R, Takenaka Y, Taki K, Tammoja K, Tan SL, Tang S, Taylor MS, Tegner J, Teichmann SA, Ueda HR, van Nimwegen E, Verardo R, Wei 
CL, Yagi K, Yamanishi H, Zabarovsky E, Zhu S, Zimmer A, Hide W, Bult C, Grimmond SM, Teasdale RD, Liu ET, Brusic V, Quackenbush J, Wahlestedt C, Mattick JS, Hume DA, Kai C, Sasaki D, Tomaru Y, Fukuda S, Kanamori-Katayama M, Suzuki M, Aoki J, Arakawa T, Iida J, Imamura K, Itoh M, Kato T, Kawaji H, Kawagashira N, Kawashima T, Kojima M, Kondo S, Konno H, Nakano K, Ninomiya N, Nishio T, Okada M, Plessy C, Shibata K, Shiraki T, Suzuki S, Tagami M, Waki K, Watahiki A, Okamura-Oho Y, Suzuki H, Kawai J, Hayashizaki Y, Consortium F, Group RGER and Genome Science G (2005) The transcriptional landscape of the mammalian genome. Science. doi:10.1126/science. 1112014

Cheng MF (2013) Hypothalamic neurogenesis in the adult brain. Front Neuroendocrinol. doi:10.1016/j.yfrne.2013.05.001

Clark BS, Blackshaw S (2014) Long non-coding RNA-dependent transcriptional regulation in neuronal development and disease. Front Genet. doi:10.3389/fgene.2014.00164

Crepin D, Benomar Y, Riffault L, Amine H, Gertler A, Taouis M (2014) The over-expression of miR-200a in the hypothalamus of ob/ob mice is linked to leptin and insulin signaling impairment. Mol Cell Endocrinol. doi:10.1016/j.mce.2013.12.016

De Leeuw F, Zhang T, Wauquier C, Huez G, Kruys V, Gueydan C (2007) The cold-inducible RNA-binding protein migrates from the nucleus to cytoplasmic stress granules by a methylationdependent mechanism and acts as a translational repressor. Exp Cell Res. doi:10.1016/j.yexcr.2007.09.017

Ellis BC, Graham LD, Molloy PL (2014) CRNDE, a long non-coding RNA responsive to insulin/IGF signaling, regulates genes involved in central metabolism. Biochim Biophys Acta 1843(2):372-386. doi:10.1016/j.bbamcr.2013.10.016

Fassan M, Sachsenmeir K, Rugge M, Baffa R (2011) Role of miRNA in distinguishing primary brain tumors from secondary tumors metastatic to the brain. Frontiers Biosci 3:970-979

Franceschini A, Szklarczyk D, Frankild S, Kuhn M, Simonovic M, Roth A, Lin J, Minguez P, Bork P, von Mering C, Jensen LJ (2013) STRING v91: protein-protein interaction networks, with increased coverage and integration. Nucleic Acids Res. doi:10. 1093/nar/gks1094

Fu C, Xi L, McCarter R, Hickey M, Han ES (2006) Early hypothalamic response to age-dependent gene expression by calorie restriction. Neurobiol Aging. doi:10.1016/j.neurobiola ging.2005.06.006

Gellert P, Ponomareva Y, Braun T, Uchida S (2013) Noncoder: a web interface for exon array-based detection of long non-coding RNAs. Nucleic Acids Res. doi:10.1093/nar/gks877

Good DJ (2010) Transcriptional regulation of sensed energy availability within hypothalamic neurons. Open Neuroendocrinol J 3:38-44

Hellwig S, Bass BL (2008) A starvation-induced noncoding RNA modulates expression of Dicer-regulated genes. Proc Natl Acad Sci USA 105(35):12897-12902. doi:10.1073/pnas.0805118105

Holden T, Nguyen A, Lin E, Cheung E, Dehipawala S, Ye J, Tremberger G Jr, Lieberman D, Cheung T (2013) Exploratory bioinformatics study of lncRNAs in Alzheimer's disease mRNA sequences with application to drug development. Comput Math Methods Med. doi:10.1155/2013/579136

Inoue $\mathrm{N}$, Yahagi $\mathrm{N}$, Yamamoto $\mathrm{T}$, Ishikawa $\mathrm{M}$, Watanabe $\mathrm{K}$, Matsuzaka T, Nakagawa Y, Takeuchi Y, Kobayashi K, Takahashi A, Suzuki H, Hasty AH, Toyoshima H, Yamada N, Shimano H (2008) Cyclin-dependent kinase inhibitor, p21WAF1/CIP1, is involved in adipocyte differentiation and hypertrophy, linking to obesity, and insulin resistance. J Biol Chem. doi:10.1074/jbc.M801824200

Kameswaran V, Kaestner KH (2014) The Missing lnc(RNA) between the pancreatic beta-cell and diabetes. Front Genet. doi:10.3389/ fgene. 2014.00200
Kokoeva MV, Yin H, Flier JS (2005) Neurogenesis in the hypothalamus of adult mice: potential role in energy balance. Science. doi: $10.1126 /$ science. 1115360

Kornfeld JW, Bruning JC (2014) Regulation of metabolism by long, non-coding RNAs. Front Genet 5:57. doi:10.3389/fgene.2014. 00057

Kung JT, Colognori D, Lee JT (2013) Long noncoding RNAs: past, present, and future. Genetics 193(3):651-669. doi:10.1534/ genetics.112.146704

Liang L, Ai L, Qian J, Fang JY, Xu J (2015) Long noncoding RNA expression profiles in gut tissues constitute molecular signatures that reflect the types of microbes. Sci Rep. doi:10.1038/srep11763

Lin N, Chang KY, Li Z, Gates K, Rana ZA, Dang J, Zhang D, Han T, Yang CS, Cunningham TJ, Head SR, Duester G, Dong PD, Rana TM (2014) An evolutionarily conserved long noncoding RNA TUNA controls pluripotency and neural lineage commitment. Mol Cell 53(6):1005-1019. doi:10.1016/j.molcel.2014.01.021

Liu S, Sheng L, Miao H, Saunders TL, MacDougald OA, Koenig RJ, Xu B (2014) SRA gene knockout protects against diet-induced obesity and improves glucose tolerance. J Biol Chem. doi:10. 1074/jbc.M114.564658

Maglich JM, Watson J, McMillen PJ, Goodwin B, Willson TM, Moore JT (2004) The nuclear receptor CAR is a regulator of thyroid hormone metabolism during caloric restriction. J Biol Chem. doi:10.1074/jbc.M313601200

Martinelli R, Nardelli C, Pilone V, Buonomo T, Liguori R, Castano I, Buono P, Masone S, Persico G, Forestieri P, Pastore L, Sacchetti L (2010) miR-519d overexpression is associated with human obesity. Obesity. doi:10.1038/oby.2009.474

Mattson MP (2005) Energy intake, meal frequency, and health: a neurobiological perspective. Annu Rev Nutr. doi:10.1146/ annurev.nutr.25.050304.092526

Mattson MP, Wan R (2005) Beneficial effects of intermittent fasting and caloric restriction on the cardiovascular and cerebrovascular systems. J Nutr Biochem. doi:10.1016/j.jnutbio.2004.12.007

McNeill E, Van Vactor D (2012) MicroRNAs shape the neuronal landscape. Neuron. doi:10.1016/j.neuron.2012.07.005

Meister B, Herzer S, Silahtaroglu A (2013) MicroRNAs in the Hypothalamus. Neuroendocrinology. doi:10.1159/000355619

Nakatsuka A, Wada J, Hida K, Hida A, Eguchi J, Teshigawara S, Murakami K, Kanzaki M, Inoue K, Terami T, Katayama A, Ogawa D, Kagechika H, Makino H (2012) RXR antagonism induces G0/G1 cell cycle arrest and ameliorates obesity by upregulating the p53-p21(Cip1) pathway in adipocytes. J Pathol. doi: $10.1002 /$ path.3001

Nogales-Cadenas R, Carmona-Saez P, Vazquez M, Vicente C, Yang X, Tirado F, Carazo JM, Pascual-Montano A (2009) GeneCodis: interpreting gene lists through enrichment analysis and integration of diverse biological information. Nucleic Acids Res. doi:10.1093/nar/gkp416

Olsen L, Klausen M, Helboe L, Nielsen FC, Werge T (2009) MicroRNAs show mutually exclusive expression patterns in the brain of adult male rats. PLoS ONE. doi:10.1371/journal.pone. 0007225

Paulsen SJ, Larsen LK, Jelsing J, Janssen U, Gerstmayer B, Vrang N (2009) Gene expression profiling of individual hypothalamic nuclei from single animals using laser capture microdissection and microarrays. J Neurosci Methods. doi:10.1016/j.jneumeth. 2008.09.024

Poplawski MM, Mastaitis JW, Yang XJ, Mobbs CV (2010) Hypothalamic responses to fasting indicate metabolic reprogramming away from glycolysis toward lipid oxidation. Endocrinology. doi:10.1210/en.2010-0702

Qureshi IA, Mehler MF (2013) Long non-coding RNAs: novel targets for nervous system disease diagnosis and therapy. Neurotherapeutics. doi:10.1007/s13311-013-0199-0 
Rinn JL, Chang HY (2012) Genome regulation by long noncoding RNAs. Annu Rev Biochem. doi:10.1146/annurev-biochem051410-092902

Roshan R, Ghosh T, Scaria V, Pillai B (2009) MicroRNAs: novel therapeutic targets in neurodegenerative diseases. Drug Discov Today. doi:10.1016/j.drudis.2009.09.009

Roy M, Kim N, Kim K, Chung WH, Achawanantakun R, Sun Y, Wayne R (2013) Analysis of the canine brain transcriptome with an emphasis on the hypothalamus and cerebral cortex. Mamm Genome. doi:10.1007/s00335-013-9480-0

Sangiao-Alvarellos S, Pena-Bello L, Manfredi-Lozano M, TenaSempere M, Cordido F (2014) Perturbation of hypothalamic microRNA expression patterns in male rats after metabolic distress: impact of obesity and conditions of negative energy balance. Endocrinology. doi:10.1210/en.2013-1770

Schneeberger M, Altirriba J, Garcia A, Esteban Y, Castano C, GarciaLavandeira M, Alvarez CV, Gomis R, Claret M (2012) Deletion of miRNA processing enzyme Dicer in POMC-expressing cells leads to pituitary dysfunction, neurodegeneration and development of obesity. Mol Metab. doi:10.1016/j.molmet.2012.10.001

Smith CM, Finger JH, Kadin JA, Richardson JE, Ringwald M (2014) The gene expression database for mouse development (GXD): putting developmental expression information at your fingertips. Dev Dyn. doi:10.1002/dvdy.24155

Sohal RS, Weindruch R (1996) Oxidative stress, caloric restriction, and aging. Science 273(5271):59-63

Sun L, Xie H, Mori MA, Alexander R, Yuan B, Hattangadi SM, Liu Q, Kahn CR, Lodish HF (2011) Mir193b-365 is essential for brown fat differentiation. Nat Cell Biol. doi:10.1038/ncb2286

Sun L, Goff LA, Trapnell C, Alexander R, Lo KA, Hacisuleyman E, Sauvageau M, Tazon-Vega B, Kelley DR, Hendrickson DG, Yuan B, Kellis M, Lodish HF, Rinn JL (2013) Long noncoding RNAs regulate adipogenesis. Proc Natl Acad Sci USA. doi:10. 1073/pnas. 1222643110

Szklarczyk D, Franceschini A, Kuhn M, Simonovic M, Roth A, Minguez P, Doerks T, Stark M, Muller J, Bork P, Jensen LJ, von Mering C (2011) The STRING database in 2011: functional interaction networks of proteins, globally integrated and scored. Nucleic Acids Res 39(Database issue):D561-D568. doi:10.1093/ nar/gkq973

Szklarczyk K, Korostynski M, Golda S, Solecki W, Przewlocki R (2012) Genotype-dependent consequences of traumatic stress in four inbred mouse strains. Genes Brain Behav. doi:10.1111/j. 1601-183X.2012.00850.x

Tabas-Madrid D, Nogales-Cadenas R, Pascual-Montano A (2012) GeneCodis3: a non-redundant and modular enrichment analysis tool for functional genomics. Nucleic Acids Res. doi:10.1093/ nar/gks402

Takanabe R, Ono K, Abe Y, Takaya T, Horie T, Wada H, Kita T, Satoh N, Shimatsu A, Hasegawa K (2008) Up-regulated expression of microRNA-143 in association with obesity in adipose tissue of mice fed high-fat diet. Biochem Biophys Res Commun. doi:10.1016/j.bbrc.2008.09.050

Tinkum KL, White LS, Marpegan L, Herzog E, Piwnica-Worms D, Piwnica-Worms H (2013) Forkhead box O1 (FOXO1) protein, but not $\mathrm{p} 53$, contributes to robust induction of $\mathrm{p} 21$ expression in fasted mice. J Biol Chem. doi:10.1074/jbc.M113.494328

Vella KR, Burnside AS, Brennan KM, Good DJ (2007) Expression of the hypothalamic transcription factor Nhlh2 is dependent on energy availability. J Neuroendocrinol. doi:10.1111/j.1365-2826. 2007.01556.x
Vijayaraghavan S, Hitman GA, Kopelman PG (1994) ApolipoproteinD polymorphism: a genetic marker for obesity and hyperinsulinemia. J Clin Endocrinol Metab. doi:10.1210/jcem.79.2.7913935

Wessel J, Chu AY, Willems SM, Wang S, Yaghootkar H, Brody JA, Dauriz M, Hivert MF, Raghavan S, Lipovich L, Hidalgo B, Fox K, Huffman JE, An P, Lu Y, Rasmussen-Torvik LJ, Grarup N, Ehm MG, Li L, Baldridge AS, Stancakova A, Abrol R, Besse C, Boland A, Bork-Jensen J, Fornage M, Freitag DF, Garcia ME, Guo X, Hara K, Isaacs A, Jakobsdottir J, Lange LA, Layton JC, Li M, Hua Zhao J, Meidtner K, Morrison AC, Nalls MA, Peters MJ, Sabater-Lleal M, Schurmann C, Silveira A, Smith AV, Southam L, Stoiber MH, Strawbridge RJ, Taylor KD, Varga TV, Allin KH, Amin N, Aponte JL, Aung T, Barbieri C, Bihlmeyer NA, Boehnke M, Bombieri C, Bowden DW, Burns SM, Chen Y, Chen YD, Cheng CY, Correa A, Czajkowski J, Dehghan A, Ehret GB, Eiriksdottir G, Escher SA, Farmaki AE, Franberg M, Gambaro G, Giulianini F, Goddard WA, 3rd, Goel A, Gottesman O, Grove ML, Gustafsson S, Hai Y, Hallmans G, Heo J, Hoffmann P, Ikram MK, Jensen RA, Jorgensen ME, Jorgensen T, Karaleftheri M, Khor CC, Kirkpatrick A, Kraja AT, Kuusisto J, Lange EM, Lee IT, Lee WJ, Leong A, Liao J, Liu C, Liu Y, Lindgren CM, Linneberg A, Malerba G, Mamakou V, Marouli E, Maruthur NM, Matchan A, McKean-Cowdin R, McLeod O, Metcalf GA, Mohlke KL, Muzny DM, Ntalla I, Palmer ND, Pasko D, Peter A, Rayner NW, Renstrom F, Rice K, Sala CF, Sennblad B, Serafetinidis I, Smith JA, Soranzo N, Speliotes EK, Stahl EA, Stirrups K, Tentolouris N, Thanopoulou A, Torres M, Traglia M, Tsafantakis E, Javad S, Yanek LR, Zengini E, Becker DM, Bis JC, Brown JB, Cupples LA, Hansen T, Ingelsson E, Karter AJ, Lorenzo C, Mathias RA, Norris JM, Peloso GM, Sheu WH, Toniolo D, Vaidya D, Varma R, Wagenknecht LE, Boeing H, Bottinger EP, Dedoussis G, Deloukas P, Ferrannini E, Franco $\mathrm{OH}$, Franks PW, Gibbs RA, Gudnason V, Hamsten A, Harris TB, Hattersley AT, Hayward C, Hofman A, Jansson JH, Langenberg C, Launer LJ, Levy D, Oostra BA, O'Donnell CJ, O'Rahilly S, Padmanabhan S, Pankow JS, Polasek O, Province MA, Rich SS, Ridker PM, Rudan I, Schulze MB, Smith BH, Uitterlinden AG, Walker M, Watkins H, Wong TY, Zeggini E, Consortium EP-I, Laakso M, Borecki IB, Chasman DI, Pedersen O, Psaty BM, Tai ES, van Duijn CM, Wareham NJ, Waterworth DM, Boerwinkle E, Kao WH, Florez JC, Loos RJ, Wilson JG, Frayling TM, Siscovick DS, Dupuis J, Rotter JI, Meigs JB, Scott RA and Goodarzi MO (2015) Low-frequency and rare exome chip variants associate with fasting glucose and type 2 diabetes susceptibility. Nat Commun. doi:10.1038/ncomms6897

Xu S, Chen P, Sun L (2015) Regulatory networks of non-coding RNAs in brown/beige adipogenesis. Biosci Rep. doi:10.1042/ BSR20150155

Yan L, Park JY, Dillinger JG, De Lorenzo MS, Yuan C, Lai L, Wang C, Ho D, Tian B, Stanley WC, Auwerx J, Vatner DE, Vatner SF (2012) Common mechanisms for calorie restriction and adenylyl cyclase type 5 knockout models of longevity. Aging Cell. doi:10. 1111/acel.12013

Yoon JH, Abdelmohsen K, Gorospe M (2013) Posttranscriptional gene regulation by long noncoding RNA. J Mol Biol. doi:10. 1016/j.jmb.2012.11.024

You LH, Zhu LJ, Yang L, Shi CM, Pang LX, Zhang J, Cui XW, Ji CB, Guo XR (2015) Transcriptome analysis reveals the potential contribution of long noncoding RNAs to brown adipocyte differentiation. Mol Genet Genomics. doi:10.1007/s00438-0151026-6 